\title{
Bloch oscillations of multidimensional dark soliton wave packets and light bullets
}

\author{
Rodislav Driben ${ }^{1 *}$, Xuekai Ma ${ }^{1}$, Stefan Schumacher ${ }^{1,2}$, and Torsten Meier ${ }^{1}$ \\ ${ }^{1}$ Department of Physics and CeOPP, University of Paderborn, Warburger Str. 100, D-33098 Paderborn, Germany \\ ${ }^{2}$ College of Optical Sciences, University of Arizona, Tucson, AZ 85721, USA \\ *Corresponding author: driben@mail.uni-paderborn.de \\ Compiled December 13, 2018
}

\begin{abstract}
The robust propagation of dark solitonic waves featuring Bloch oscillations (BOs) in media with a Kerr nonlinearity is demonstrated. The models considered have a discrete refractive index gradient in one dimension and are continuous in the orthogonal direction or directions. Such systems can be realized in photonic systems, where temporal dispersion of normal type is able to support dark solitons. The demonstrated effects may also appear in the dynamics of Bose-Einstein condensates (BEC) where dark solitons appear due to the joint action of diffraction and a self-defocusing nonlinearity. Our analysis shows furthermore that a periodic variation of the refractive index gradient in the propagation direction allows to realize the spatial analogue of dynamical localization. In addition, we demonstrate that dark solitons serve as excellent carriers for light bullets of peculiar dark-bright type that can also feature robust BOs. ๑ 2018 Optical Society of America
\end{abstract}

OCIS codes: $\quad$ (190.4370) Nonlinear optics, fibers; (190.5530) Pulse propagation and temporal soliton; (190.6135) Spatial solitons; (260.5950) Self-focusing

http://dx.doi.org/10.1364/ao.XX.XXXXXX

90 years after their initial prediction in the seminal papers of Bloch and Zener [1,2] BOs are still a topic of tremendous current scientific interest. Following an intense theoretical debate about their actual existence in the solid state community, BOs were first observed experimentally using four-wave mixing in electrically-biased semiconductor superlattices in 1992 [3]. Soon thereafter BOs were also observed in other systems, namely atoms in optical lattices [4] and also in photonics using coupled waveguides [5, 6]. Until very recently, however, Zener's original conception of spatio-temporally oscillating wave packets was not directly observed with cold atoms but rather inferred from measurements of the dynamics in momentum space. This gap was closed by the observation of position-space BOs in an ultracold gas by directly imaging the full phase-space evolution during BOs which also allowed to image the band structure of the ground and excited state Bloch bands [7]. The ability to experimentally detect BOs in real space can be a strong asset to quantum metrology and spatiotemporal control as well as for atom-interferometric force sensing and gradiometry $[8,9]$. Another even more straightforward possibility is the application of the full phase-space evolution to study complex band structures such as higher-dimensional lattices, Floquet-hybridized bands, and topologically nontrivial bands [10].

In photonic settings several studies analyzed BOs in the pres- ence of a Kerr nonlinearity. However, in models with only discrete diffraction the nonlinearity leads to a rapid spreading of initially localized wave packets and thus a purely destructive influence on the BO dynamics. On the other hand, it was shown in recent works $[11,12]$ that adding another dimension with continuous diffraction may lead to a constructive effect of the nonlinearity, i.e., a localization of the wave packet in space and the creation of a quasi-solitonic regime of propagation showing robust BOs.

Dark solitons exist in the normal dispersion regime and they are characterized by a dip on top of a flat intensity background [13]. Also vectorial dark solitons [14] as well as coupled bright and dark solitons $[15,16]$ and the dynamics of dark solitons in presence of higher order dispersive effects $[17,18]$ were discovered and studied. Several works addressed the interaction of dispersive waves with dark solitons [17-19] and recently dark soliton dynamics was demonstrated in exciton polaritons [20, 21].

In this letter, we investigate nonlinear photonic systems which have a discrete refractive index gradient in one dimension and are continuous in the orthogonal direction or directions. We demonstrate the robust propagation of dark solitonic waves featuring BOs in media with a Kerr nonlinearity. In the considered systems a periodic variation of the refractive index gradient in the propagation direction allows to realize the spatial analogue 

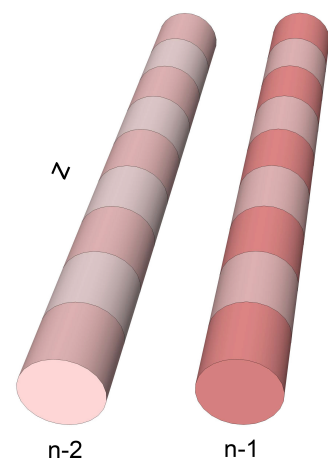

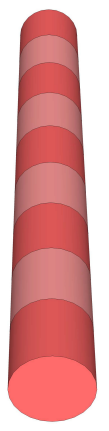

$\mathrm{n}$

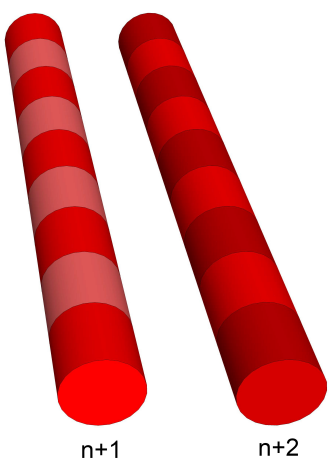

Fig. 1. (Color online) Schematic illustration of a waveguide array with a linear gradient in the n-direction represented by the color saturation which may depend on the position in the propagation direction $z$. Physically the gradient and its $\mathrm{z}$-dependence can be realized by varying the refractive index in space.

of dynamical localization. In addition, we demonstrate that dark solitons serve as excellent carriers for light bullets of peculiar dark-bright type and predict robust BOs in their propagation.

For our investigations we adopt the model used in [22]. The system is continuous in time $t$, discrete along the coordinate $n$, and the evolution coordinate is the propagation direction $z$. Due to the rich variety of nonlinear phenomena this mixed discretecontinuous model has been studied intensively, see, e.g., [23] and references therein. Mathematically, such a photonic system is described by the equation

$$
\begin{array}{r}
-i \partial_{z} \psi_{n}=\beta \frac{\partial^{2} \psi_{n}}{\partial t^{2}}+\sigma\left(\psi_{n+1}+\psi_{n-1}-2 \psi_{n}\right)+ \\
+\alpha\left|\psi_{n}\right|^{2} \psi_{n}+\gamma(z) n \psi_{n}
\end{array}
$$

Here, $\psi_{n}(z, t)$ is the field, $\beta$ is the coefficient of the leading dispersion term that can be either positive for the anomalous dispersion or negative for normal dispersion, $\sigma$ is the coupling between neighboring waveguides of the array, $\gamma(z)$ is the strength of the linear gradient which may depend on the propagation coordinate, and $\alpha$ represents the nonlinearity. This system is schematically presented in Fig. 1. The model is also applicable for the description of BEC with evolution in time rather than along the spatial coordinate and both the continuous and the discrete axes being spatial [11]. For the case of BECs the term with the second derivative represents spatial diffraction forcing $\beta$ to take only positive values, however, the nonlinearity coefficient $\alpha$ can have both signs. Thus in the photonic realization we can expect dark solitons for negative values $\beta$ acting together with positive values of $\alpha$, while for BECs we expect dark solitons for positive values of $\alpha$ and a self-defocusing nonlinearity represented by negative values of $\alpha$.

Following the analysis of [11] we start from the approximate analytical solution for the hybrid wave packet and thus launch wave packets of the following profile

$$
\psi_{n}^{(z=0)}(t)=A(n) \tanh [A(n) t] .
$$

with a Gaussian envelope

$$
A(n)=a_{0} \exp \left(-n^{2} / w^{2}\right) .
$$

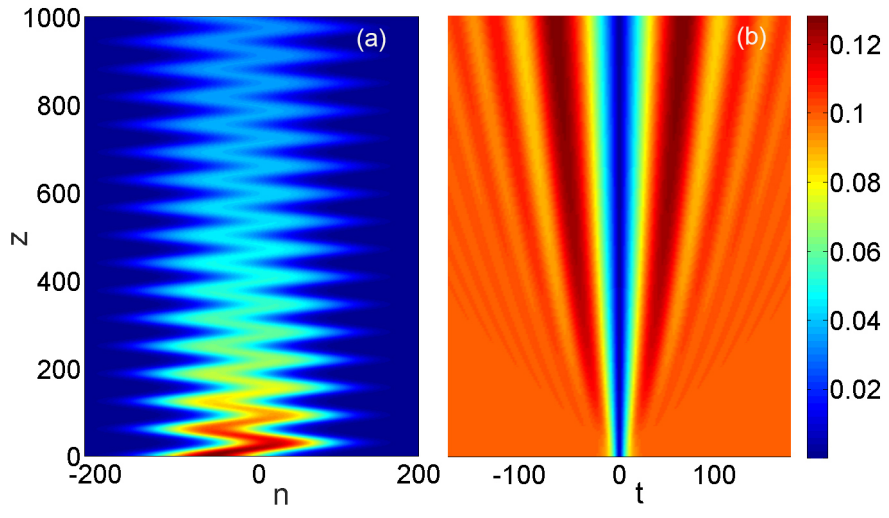

Fig. 2. (Color online) Evolution of the wave packet in the linear regime, i.e., with $\alpha=0$ in Eq. (1), in the $(n, z)$ (a) and $(t, z)$ (b) planes, respectively. For better visibility of the low intensity regions of wave packets hereafter we show moduli of the fields instead of intensities (moduli squared).
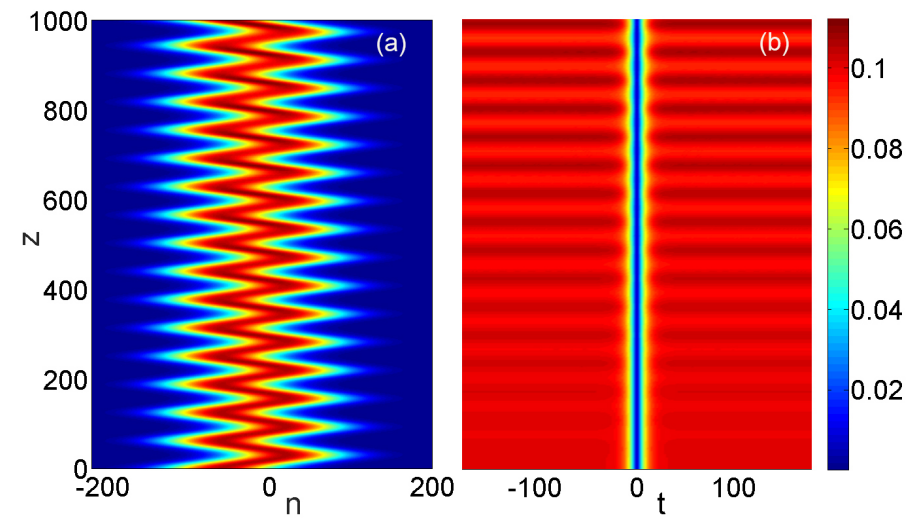

Fig. 3. (Color online) Evolution of the wave packet in the nonlinear regime, i.e., with $\alpha=1$ and $a_{0}=0.1 \mathrm{in} \mathrm{Eq.} \mathrm{(1),} \mathrm{in} \mathrm{the}$ $(n, z)(\mathrm{a})$ and $(t, z)(\mathrm{b})$ planes, respectively.

Here, we restrict the analysis to the case of relatively long pulses for which high-order dispersion and high-order nonlinear effects can be neglected. In our numerical simulations we use the values $\sigma=2, \beta=-0.5$, and $\alpha=1$ in the nonlinear regime and set $\alpha=0$ for the linear case. The gradient strength is $\gamma=0.1$ and we vary it with the propagation coordinate $\mathrm{z}$ only for the demonstration of the dynamical localization. The parameters of the initial pulse are chosen as $a_{0}=0.1$ and $w=100$.

Results of numerical simulations illustrating the dynamics of the system in the linear regime $(\alpha=0)$ are presented in Fig. 2 . As can be clearly seen the dark soliton wave packet experiences only very few BOs in n-z plane due to the rapid broadening induced by the dispersion in the $\mathrm{t}-\mathrm{z}$ plane. The situation drastically improves in the nonlinear regime $(\alpha=1)$ where we use temporal dark solitons as the "carriers" for the wave as demonstrated in Fig. 3. Due to counteracting effects of the nonlinearity and the dispersion [11] a very effective localization in both the $t-z$ and in $n-z$ planes is achieved and a regime of very robust BOs is obtained. A further increase of the strength of the nonlinear regime leads to a splitting of $\mathrm{BOs}$ as it was also observed in the case of bright solitons [11]; see the simulation result with a doubled input amplitude of $a_{0}=0.2$ presented in Fig. 4 . 

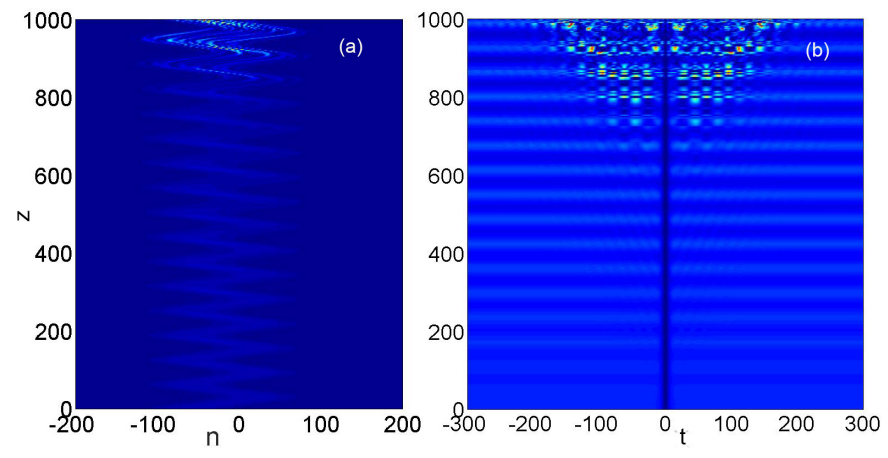

Fig. 4. (Color online) Evolution of the wave packet in the nonlinear regime, i.e., with $\alpha=1$ and $a_{0}=0.2$ in Eq. (1), in the $(n, z)(\mathrm{a})$ and $(t, z)(\mathrm{b})$ planes, respectively.
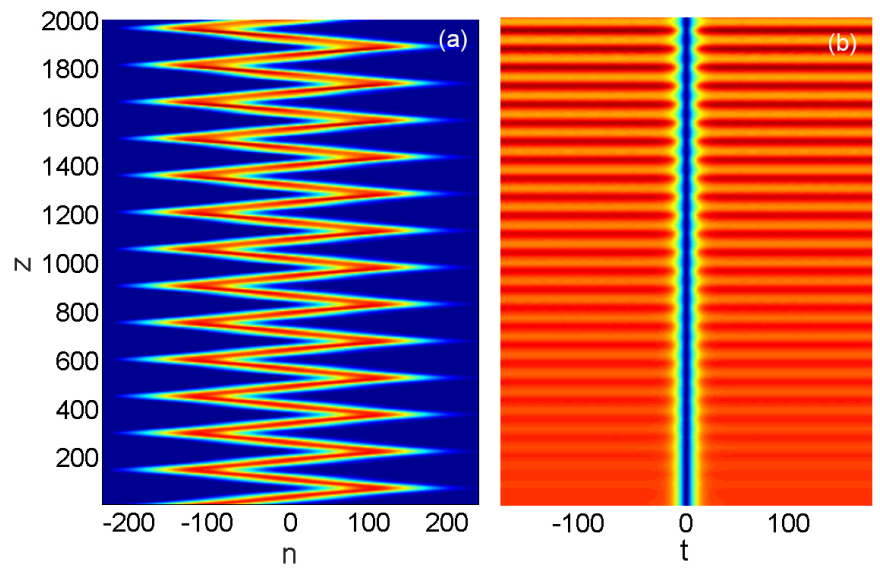

Fig. 5. (Color online) Long-time evolution of the wave packet in the nonlinear regime in the $n-z$ and in the $t-z$ planes, respectively. (a) and (b) dynamical localization of the wave packet driven with a strength and frequency corresponding to the first root of the Bessel function $J_{0}$. The parameters are chosen as $\gamma=0.1$ and $\omega=0.0416$. The input is launched with $a_{0}=0.1$ and $w=100$.

Also the spatial analogue of dynamical localization [26, 27] can be realized with dark solitons. For these simulations we alternate the gradient strength periodically as function of the propagation coordinate as

$$
\gamma(z)=\gamma_{0} \cos (\omega z)
$$

Dynamical localization, i.e., the complete suppression of the coupling $\sigma$ between the waveguides, is obtained for

$$
J_{0}\left(\gamma_{0} / \omega\right)=0,
$$

i.e., the ratio between the strength and the spatial frequency of the gradient must equal a root of the Bessel function $J_{0}[26,27]$. As demonstrated in Fig. 5, for the case of $\gamma_{0} / \omega=2.404$ which is very close to the first zero of $J_{0}$, the dark soliton wave packet oscillates in the $(n, z)$ plane with the spatial period $2 \pi / \omega \approx 151$ and remains localized in both the $(n, z)$ and the $(t, z)$ planes during the propagation.

Adding another continuous dimension, that is diffraction along the $x$-axis, provides the intriguing possibility to realize a light bullet of mixed dark-bright nature. The governing equation
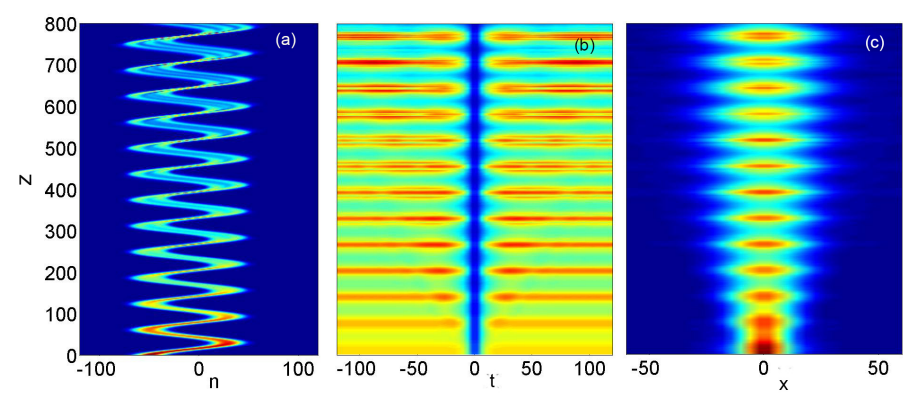

Fig. 6. (Color online) Evolution of a multidimensional light bullet of mixed dark-bright type, with $\alpha=1$ and $a_{0}=0.1$ in Eq. (6), in the $(n, z)(\mathrm{a})$, the $(t, z)(\mathrm{b})$, and , the $(x, z)$ planes, respectively.

is similar to Eq. (1) but with the addition of a second derivative term with respect to $x$ representing spatial diffraction

$$
\begin{array}{r}
-i \partial_{z} \psi_{n}=D \frac{\partial^{2} \psi_{n}}{\partial x^{2}}+\beta \frac{\partial^{2} \psi_{n}}{\partial t^{2}}+\sigma\left(\psi_{n+1}+\psi_{n-1}-2 \psi_{n}\right)+ \\
+\alpha\left|\psi_{n}\right|^{2} \psi_{n}+\gamma(z) n \psi_{n} .
\end{array}
$$

Here, D stands for the diffraction coefficient and we take $\mathrm{D}=0.5$ without loss of generality. Physically this model represents waveguide arrays having a finite depth rather than arrays of thin stripes. As shown in Fig. 6, such a hybrid light bullets carrying BOs propagate in a very robust manner for many BO cycles.

In summary, we have shown that joint action of a selffocusing nonlinearity, a refractive index gradient, and normal temporal dispersion is able to support robust hybrid waves, which feature BOs of dark solitons in properly designed multidimensional photonic systems. Such oscillations can be observed even for moderate nonlinearities and large enough values of the linear potential, when the band-gap picture of the underlying linear lattice is not applicable anymore. We also demonstrate that the nonlinearity plays a constructive role in supporting the robustness of dynamical localization which appears for a periodic alternation of the gradient strength. The predicted effects are also realizable in the dynamics of BECs. Moreover, we demonstrate robust propagation of peculiar light bullet objects having both features of BOs and dark-bright solitons.

\section{FUNDING}

We acknowledge support by the Deutsche Forschungsgemeinschaft (DFG, German Research Foundation) - project number 231447078 - TRR 142 (project A04). RD and TM thank the PC 2 (Paderborn Center for Parallel Computing) for providing computing time. SS also acknowledges support through the DFG Heisenberg programme - project number 270619725. XM acknowledges support from the NSFC (Grant No. 11804064).

\section{REFERENCES}

1. F. Bloch, Z. Phys. 52, 555 (1928).

2. C. Zener,Proc. R. Soc. A 145, 523 (1934).

3. J. Feldmann, K.Leo, J.Shah, D. B. A. Miller, J. E. Cunningham, S. Schmitt-Rink, T. Meier, G. von Plessen, A. Schulze, and P. Thomas, Phys. Rev. B 46, 7252 (1992).

4. M. B. Dahan, E. Peik, J. Reichel, Y. Castin, and C. Salomon, Phys. Rev. Lett. 76, 4508 (1996).

5. U. Peschel, T. Pertsch, and F. Lederer, Opt. Lett. 23, 1701 (1998). 
6. R. Morandotti, U. Peschel, J. S. Aitchison, H. S. Eisenberg, and Y. Silberberg, Phys. Rev. Lett. 83, 4756 (1999).

7. Z. A. Geiger, K. M. Fujiwara, K. Singh, R. Senaratne, S. V Rajagopal, M. Lipatov, T. Shimasaki, R. Driben, V. V. Konotop, T. Meier, and D. M. Weld, Phys. Rev. Lett. 120, 213201 (2018).

8. I. Carusotto, L. Pitaevskii, S. Stringari, G. Modugno, and M. Inguscio, Phys. Rev. Lett. 95, 093202 (2005).

9. B. Estey, C. Yu, H. Müller, P.-C. Kuan, and S.-Y. Lan, Phys. Rev. Lett. 115, 083002 (2015).

10. M. Holthaus, Journal of Physics B 49, 013001 (2016).

11. R. Driben, V. V. Konotop, T. Meier, and A. V. Yulin, Scientific Reports 7, 3194 (2017)

12. A. Yulin, R. Driben, and T. Meier, Phys. Rev. A 96, 033827 (2017).

13. G. P. Agrawal, Nonlinear Fiber Optics, 4th ed. (Academic Press, Boston 2007)

14. Y.S Kivshar, S.K Turitsyn, Opt. Lett. 18, 337-339 (1993)

15. V. V. Afanasjev, Y. S. Kivshar, V. V. Konotop, and V. N. Serkin, Opt. Lett. 14, 805 (1989)

16. S. Trillo, S. Wabnitz, E. M. Wright, and G. I. Stegeman, Opt. Lett. 13, 871 (1988)

17. V. V. Afanasjev, Y. S. Kivshar, and C. R. Menyuk, Opt. Lett. 21, 1975 (1996)

18. C. Milián, D. V. Skryabin, and A. Ferrando, Opt. Lett. 34(14), 2096 (2009)

19. I. Oreshnikov, R. Driben, A.V. Yulin, Opt. Lett. 40 (21), 4871 (2015)

20. X Ma, OA Egorov, S Schumacher, Phys. Rev. Lett. 118 (15), 157401 (2017)

21. P. M Walker et. al, Phys. Rev. Lett. 119 (9), 097403 (2017)

22. A. B. Aceves, G. G. Luther, C. De Angelis, A. M. Rubenchik, and S. K. Turitsyn, Phys. Rev. Lett. 75, 73 (1995).

23. B. A. Malomed, D. Mihalache, F. Wise, and L. Torner, J. Optics B: Quant. Semicl. Opt. 7, R53 (2005).

24. G. Fibich and B. Ilan, Opt. Lett. 29, 887 (2004).

25. A.V. Yulin, D.V. Skryabin, and P.St.J. Russell, Opt. Lett. 30, 525 (2005).

26. D. H. Dunlap and V. M. Kenkre, Phys. Rev. B 34, 3625 (1986).

27. M. Holthaus, Phys. Rev. Lett. 69, 351 (1992).

\section{REFERENCES}

1. F. Bloch, Über die Quantenmechanik der Elektronen in Kristallgittern, Z. Phys. 52, 555 (1928).

2. C. Zener, A theory of the electrical breakdown of solid dielectrics, Proc. R. Soc. A 145, 523 (1934).

3. J. Feldmann, K.Leo, J.Shah, D. B. A. Miller, J. E. Cunningham, S. Schmitt-Rink, T. Meier, G. von Plessen, A. Schulze, and P. Thomas, Optical investigation of Bloch oscillations in a semiconductor superlattice, Phys. Rev. B 46, 7252 (1992).

4. M. B. Dahan, E. Peik, J. Reichel, Y. Castin, and C. Salomon, Bloch Oscillations of Atoms in an Optical Potential, Phys. Rev. Lett. 76, 4508 (1996).

5. U. Peschel, T. Pertsch, and F. Lederer, Optical Bloch oscillations in waveguide arrays, Opt. Lett. 23, 1701 (1998).

6. R. Morandotti, U. Peschel, J. S. Aitchison, H. S. Eisenberg, and Y. Silberberg, Experimental observation of linear and nonlinear optical Bloch oscillations, Phys. Rev. Lett. 83, 4756 (1999).

7. Z. A. Geiger, K. M. Fujiwara, K. Singh, R. Senaratne, S. V Rajagopal, M. Lipatov, T. Shimasaki, R. Driben, V. V. Konotop, T. Meier, and D. M. Weld, Observation and uses of position-space Bloch oscillations in an ultracold gas, Phys. Rev. Lett. 120, 213201 (2018).

8. I. Carusotto, L. Pitaevskii, S. Stringari, G. Modugno, and M. Inguscio, Phys. Rev. Lett. 95, 093202 (2005).

9. B. Estey, C. Yu, H. Müller, P.-C. Kuan, and S.-Y. Lan, Phys. Rev. Lett. 115, 083002 (2015)

10. M. Holthaus, Journal of Physics B 49, 013001 (2016).

11. R. Driben, V. V. Konotop, T. Meier, and A. V. Yulin, Bloch oscillations sustained by nonlinearity, Sci. Rep. 7, 3194 (2017).

12. A. Yulin, R. Driben, and T. Meier, Bloch oscillations and resonant radiation of light propagating in arrays of nonlinear fibers with highorder dispersion, Phys. Rev. A 96, 033827 (2017).

13. G. P. Agrawal, Nonlinear Fiber Optics, 4th ed. (Academic Press, Boston 2007)

14. YS Kivshar, SK Turitsyn "Vector dark solitons" Opt. Lett. 18, 337-339 (1993)

15. V. V. Afanasjev, Y. S. Kivshar, V. V. Konotop, and V. N. Serkin, "Dynamics of coupled dark and bright optical solitons" Opt. Lett. 14, 805 (1989)

16. S. Trillo, S. Wabnitz, E. M. Wright, and G. I. Stegeman, "Optical solitary waves induced by cross-phase modulation" Opt. Lett. 13, 871 (1988)

17. V. V. Afanasjev, Y. S. Kivshar, and C. R. Menyuk, "Effect of third-order dispersion on dark solitons," Opt. Lett. 21, 1975-1977 (1996)

18. C. Milián, D. V. Skryabin, and A. Ferrando, "Continuum generation by dark solitons," Opt. Lett. 34(14), 2096-2098 (2009)

19. I. Oreshnikov, R. Driben, A.V. Yulin, "Weak and strong interactions between dark solitons and dispersive waves," Opt. Lett. 40 (21), 4871 (2015)

20. X Ma, OA Egorov, S Schumacher, "Creation and manipulation of stable dark solitons and vortices in microcavity polariton condensates," Phys. Rev. Lett. 118 (15), 157401 (2017)

21. PM Walker et. al, Phys. Rev. Lett. 119 (9), 097403 (2017)

22. A. B. Aceves, G. G. Luther, C. De Angelis, A. M. Rubenchik, and S. K. Turitsyn, Phys. Rev. Lett. 75, 73 (1995).

23. B. A. Malomed, D. Mihalache, F. Wise, and L. Torner, Spatiotemporal optical solitons, J. Optics B: Quant. Semicl. Opt. 7, R53 (2005).

24. G. Fibich and B. Ilan, Optical light bullets in a pure Kerr medium, Opt. Lett. 29, 887 (2004).

25. A.V. Yulin, D.V. Skryabin, and P.St.J. Russell, Resonant radiation and collapse of ultrashort pulses in planar waveguides, Opt. Lett. 30, 525 (2005).

26. D. H. Dunlap and V. M. Kenkre, Dynamic localization of a charged particle moving under the influence of an electric field, Phys. Rev. B 34, 3625 (1986).

27. M. Holthaus, Collapse of minibands in far-infrared irradiated superlattices, Phys. Rev. Lett. 69, 351 (1992). 